\title{
Finite difference-finite element approach for solving fractional Oldroyd-B equation
}

\author{
Amer Rasheed ${ }^{1 *}$, Abdul Wahab², Shaista Qaim Shah ${ }^{3}$ and Rab Nawaz ${ }^{4}$
}

\author{
"Correspondence: \\ amer.rasheed@lums.edu.pk \\ 'Department of Mathematics, \\ Lahore University of Management \\ Sciences, Opposite Sector U, DHA, \\ Lahore, 54792, Pakistan \\ Full list of author information is \\ available at the end of the article
}

\begin{abstract}
In this article, we study an unsteady flow of an anomalous Oldroyd-B fluid confined between two infinite parallel plates subject to no-slip condition at boundary. The flow is induced by a linear acceleration of the lower plate in its own plane. A standard Galerkin finite element method is adopted to construct an approximate solution blended with a finite difference approximation for Caputo fractional time derivatives. The convergence of the proposed numerical scheme is substantiated, and error estimates are provided in appropriate norms. Some adequate numerical simulations are performed in order to elucidate the dominance of characteristic flow parameters of velocity field in the prescribed configuration.
\end{abstract}

MSC: 35A15; 76A05; 76A10; 76D99; 76M10

Keywords: anomalous fluid; Oldroyd-B fluid; finite element method; stability; convergence

\section{Introduction}

The curiosity to understand mass transfer phenomena in fluids obeying non-Newtonian rheological paradigms is increasing due to broad range of engineering applications and apposite industrial processes. The examples include material plasticizing and solidification processes for manufacturing parts, oil-well drilling, and fossil fuel combustion; see, for instance, [1-3], the review article [4], and references therein. Spirited researchers endeavoring in assorted domains have been experimentally testing, mathematically modeling, establishing numerical approximations, and designing algorithms for analyzing various flow problems in different geometric and flow configurations [3-15]. Mathematicians are particularly exposed to challenging mathematical riddles, for instance, related to solvability, consistency, stability, and thermodynamic compatibility of constitutive flow models, their solutions, and approximations [1, 4, 11, 12, 16-23].

Several approximate and self-consistent non-Newtonian rheological models are proposed over the past decades as no single one can encompass assorted features of all the fluids. These models are classified into differential, rate, and integral types. The interested readers are referred, for instance, to $[20,24,25]$ for detailed accounts. In particular, the stress relaxation in polymer processing is usually predicted using rate-type fluid models such as Maxwell, Oldroyd-B, or Burgers fluids [7, 9, 11, 24, 26-29].

In certain non-Newtonian fluids, an anomalous rheological model provides a more realistic fit to the experimental data $[13,30,31]$. For instance, the anomalous Maxwell model

(c) 2016 Rasheed et al. This article is distributed under the terms of the Creative Commons Attribution 4.0 International License (http://creativecommons.org/licenses/by/4.0/), which permits unrestricted use, distribution, and reproduction in any medium, provided you give appropriate credit to the original author(s) and the source, provide a link to the Creative Commons license, and indicate if changes were made. 
yields algebraically decaying stress relaxation modulus resulting in a good agreement to experimental data [31], whereas the Brownian Maxwell fluid fails to do so, at least over complete range of frequencies. Moreover, as indicated by Bagley and Torvik [32, 33], the molecular theory is harmonic to anomalous viscoelastic models. The anomalous nature of the flow is usually modeled with fractional-order time derivatives replacing those of integer order in classical stress-strain relations. The issues concerning well-posedness and thermodynamic stability of the anomalous viscoelastic flow models have been addressed, for instance, in [23, 34, 35].

The constitutive initial-boundary value problems for non-Newtonian fluids rarely have exact and closed-form analytic solutions since these models are strongly nonlinear, whereas sufficient boundary conditions are not often available. Thus, numerical and asymptotic techniques are sought exploiting supplementary information on the flow profile. Unfortunately, the asymptotic solutions are mostly divergent for strongly nonlinear problems and large values of pertinent flow parameters such as Péclet, Reynolds, and Weissenberg numbers $[36,37]$. Therefore, great interest in numerical approximation techniques in non-Newtonian fluid mechanics is observed in recent years; see, for instance, $[14,15,19,38-40]$, among many others.

The hot topics in numerical analysis include challenging issues related to instability of approximate solutions due to strong nonlinearity, convection dominance, and parabolichyperbolic nature of non-Newtonian flow problems for increasing values of flow parameters. A variety of stabilization and numerical approximation frameworks are consequently introduced and analyzed. More recently, frameworks for approximating solutions to time and/or space fractional differential equations in connection with subdiffusion and superdiffusion, viscoelastic wave propagation, and anomalous flow problems are discussed; see [40-43] and references therein. The so-called $L 1$-finite difference approximation method is invoked together with space approximation schemes to obtain numerical solutions to time-discretized models, such as spatial finite difference, spectral, lumped mass, and Galerkin finite element techniques.

In this article, we provide a numerical exposition of flow phenomena for an incompressible anomalous Oldroyd-B fluid using a standard Galerkin finite element method (FEM) blended with finite difference approximation in time. We consider the fluid confined between two infinite parallel plates, which starts flowing due to a linear acceleration of the lower plate in its own plane, whereas the upper plate is kept rigid and no slip condition at boundaries is imposed. The anomalous behavior of the Oldroyd-B fluid is modeled with left-sided Caputo fractional time derivatives thereby generalizing the canonical Brownian Oldroyd-B fluid model that can be perceived as a limiting case. The objective of the investigation is twofold: (1) understanding the velocity profile in the aforementioned flow and (2) deploying standard Lagrange-Galerkin FEM together with the L1-finite difference scheme and subsequently performing a convergence analysis following the pioneer works in [41-43]. Albeit, the assumptions of a linear plate acceleration and negligible pressure gradient are made for brevity, and the analysis contained herein can be analogously performed otherwise. The results can be extended to the Burgers fluids and will be discussed in a forthcoming investigation.

The rest of this contribution is arranged in the following manner. In Section 2, the flow problem is mathematically formulated. The equations governing the flow are detailed 
(see Section 2.1), and the associated initial boundary value problem (IBVP) is derived and nondimensionalized (Section 2.2). The finite element approximation to the velocity field is presented in Section 3. First, a few notions and notation are collected (Section 3.1), and a finite-difference-based temporal discretization scheme is presented for the fractional time derivatives (Section 3.2). Then, the spatial discretization of the IBVP is derived using Lagrange interpolation functions (Section 3.3). The convergence analysis of the numerical scheme is performed in Section 4, and the numerical simulations are presented in Section 5. Finally, the findings of the investigation are summarized in Section 6.

\section{Formulation of flow problem}

We fix the following notation henceforth.

Definition 2.1 The left-sided Caputo fractional derivative of order $\gamma(\gamma \in \mathbb{C}, \Re e\{\gamma\}>0)$ with respect to $t$, denoted by $\frac{\partial^{\gamma}}{\partial t^{\gamma}}$ or $\partial_{t}^{\gamma}$, is defined as

$$
\partial_{t}^{\gamma} \phi(t):=\frac{1}{\Gamma(n-\gamma)} \int_{0}^{t}(t-\tau)^{n-\gamma-1} \frac{\partial^{n}}{\partial \tau^{n}} \phi(\tau) d \tau, n-1<\Re e\{\gamma\}<n, \quad n \in \mathbb{N},
$$

where $\Gamma$ is the standard Euler's gamma function given by (see, e.g., the monographs [44, 45])

$$
\Gamma(z):=\int_{\mathbb{R}} \xi^{z-1} e^{-\xi} d \xi, \quad z \in \mathbb{C}, \Re\{z\}>0 .
$$

Remark 2.2 The fractional derivative $\partial_{t}^{\gamma} \phi$ converges to the canonical integer-order derivative $\partial_{t}^{n} \phi$ as the parameter $\gamma \in \mathbb{R} \rightarrow n \in \mathbb{N}$, where $n-1<\gamma<n$ (see, e.g., [45], p.92).

The following proposition from [45], Proposition 2.16, will be useful in the sequel.

Proposition 2.3 Let $\gamma \in \mathbb{C}$ with $\Re e\{\gamma\}>0$, and $n \in \mathbb{N}$ such that $n-1<\Re e\{\gamma\}<n$. Then, for all $s \in \mathbb{C}$ with $\Re e\{s\}>n-1$,

$$
\partial_{t}^{\gamma} t^{s}:=\frac{\Gamma(s+1)}{\Gamma(s+1-\gamma)} t^{s-\gamma}, \quad \forall t>0
$$

\subsection{Flow configuration and governing equations for an ordinary fluid}

Consider the flow of an incompressible Oldroyd-B fluid between two infinite parallel plates at distance $2 L>0$ apart. Without loss of generality, the $y$-axis is taken perpendicular to the plates, whereas the planes $y=-L$ and $y=L$ represent the lower and upper plates, respectively (see Figure 1). Consider the velocity field

$$
\mathbf{U}:=u \mathbf{e}_{\boldsymbol{x}}+v \mathbf{e}_{\mathbf{y}}+w \mathbf{e}_{\mathbf{z}}
$$

where $\left\{\mathbf{e}_{\mathbf{x}}, \mathbf{e}_{\mathbf{y}}, \mathbf{e}_{\mathbf{z}}\right\}$ is the canonical basis of $\mathbb{R}^{3}$. Assume that the mainstream flow takes place only along the $x$-axis. Then, $u=u(y, t)$ and $v \equiv 0 \equiv w$. Consequently,

$$
\mathbf{U}=u(y, t) \mathbf{e}_{\boldsymbol{x}}
$$




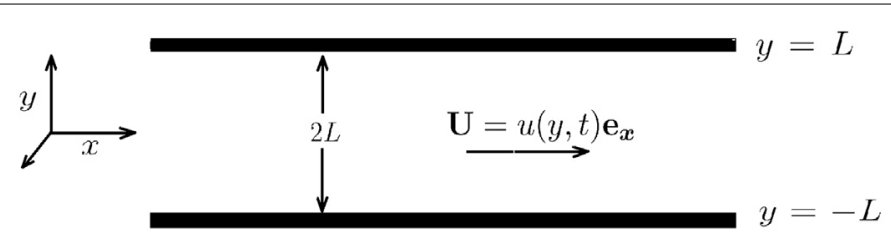

Figure 1 Geometric configuration.

Recall that the first Rivlin-Ericksen kinematic tensor $\mathbb{A}_{1}$ is defined by

$$
\mathbb{A}_{1}:=\nabla \mathbf{U}+(\nabla \mathbf{U})^{\top}
$$

where the superscript $T$ indicates the transpose operation. The relevant stress tensor, denoted by $\mathbb{T}$, is given by

$$
\mathbb{T}=-p \mathbb{I}+\mathbb{S}
$$

where $p$ is the hydrostatic pressure of the fluid, $\mathbb{I}$ is the identity tensor, and $\mathbb{S}$ is the extra stress tensor defined by the relation

$$
\left(1+\lambda_{1} \mathcal{D}_{t}\right)[\mathbb{S}]=\mu\left(1+\lambda_{2} \mathcal{D}_{t}\right)\left[\mathbb{A}_{1}\right]
$$

Here $\mu>0$ is the dynamic viscosity, $\lambda_{1}$ is the relaxation time, and $\lambda_{2}$ is the retardation time. The operator $\mathcal{D}_{t}$ is the so-called Oldroyd or upper convected derivative defined by

$$
\mathcal{D}_{t}[\mathbb{S}]:=\frac{\partial}{\partial t}[\mathbb{S}]+(\mathbf{U} \cdot \nabla)[\mathbb{S}]+(\nabla \mathbf{U}) \mathbb{S}+\mathbb{S}(\nabla \mathbf{U})^{\top}
$$

We shall also consider the extra stress tensor of the form

$$
\mathbb{S}=\mathbb{S}(y, t):=\left(\begin{array}{lll}
s_{x x} & s_{x y} & s_{x z} \\
s_{y x} & s_{y y} & s_{y z} \\
s_{z x} & s_{z y} & s_{z z}
\end{array}\right) .
$$

Moreover, for the fluid initially at rest, it is reasonable to impose the initial conditions

$$
\mathbb{S}(y, 0)=0=\frac{\partial \mathbb{S}}{\partial t}(y, 0)
$$

Remark 2.4 The thermodynamic stability, necessary condition for well-posedness in the sense of Hadamard, and causality constraints restrict the values of relaxation and retardation times $\lambda_{1}$ and $\lambda_{2}$ to be such that $0<\lambda_{2}<\lambda_{1}$ (refer, e.g., to [20,46] for further details).

We consider an incompressible fluid such that the governing equations are

$$
\begin{aligned}
& \nabla \cdot \mathbf{U}=0, \\
& \rho_{1}\left[\frac{\partial \mathbf{U}}{\partial t}+(\mathbf{U} \cdot \nabla) \mathbf{U}\right]=\nabla \cdot \mathbb{S},
\end{aligned}
$$


where $\rho_{1}>0$ is the (constant) density of the fluid. The body forces and pressure gradient are neglected for simplicity.

\subsection{Flow problem}

In this section, the constitutive equations corresponding to a fractional Oldroyd-B fluid are derived together with relevant initial and boundary conditions. Toward this end, we first briefly derive constitutive equations for the flow of a canonical Oldroyd-B fluid and then highlight appropriate changes in order to incorporate anomalous behavior of fluid rheology.

Note that the velocity field $\mathbf{U}$ in (2) automatically satisfies the equation of continuity and $(\mathbf{U} \cdot \nabla) \mathbf{U} \equiv 0$. By equations (2), (3), (5), and (6) relation (4), together with initial conditions (7), yields, for all $t>0$ and $|y|<L$,

$$
\begin{aligned}
& s_{x z}=s_{z x}=s_{y z}=s_{z y}=s_{y y}=s_{z z}=0, \\
& \left(1+\lambda_{1} \frac{\partial}{\partial t}\right)\left[s_{x x}\right]-2 \lambda_{1} s_{x y} \frac{\partial u}{\partial y}=-2 \mu \lambda_{2}\left(\frac{\partial u}{\partial y}\right)^{2}, \\
& \left(1+\lambda_{1} \frac{\partial}{\partial t}\right)\left[s_{x y}\right]=\left(1+\lambda_{2} \frac{\partial}{\partial t}\right)\left[\frac{\partial u}{\partial y}\right] .
\end{aligned}
$$

Now the momentum equation (9) for an ordinary Oldroyd-B fluid by (2) and relations (10)-(12) yields

$$
\rho_{1}\left(1+\lambda_{1} \frac{\partial}{\partial t}\right)\left[\frac{\partial u}{\partial t}\right]=\mu\left(1+\lambda_{2} \frac{\partial}{\partial t}\right)\left[\frac{\partial^{2} u}{\partial y^{2}}\right]
$$

We are interested in the flow between two infinite plates wherein the upper plate is fixed, whereas the lower plate exhibits variable acceleration for $t>0$, and the whole system is at rest initially. Therefore, we can write the boundary and initial conditions as

$$
\begin{aligned}
& u(L, t)=0 \quad \text { and } \quad u(-L, t)=A \frac{\mu^{2}}{\rho_{1}^{2} L^{4}} t^{2}, \quad t>0, \\
& u(y, 0)=0=\frac{\partial u}{\partial t}(y, 0), \quad|y| \leq L,
\end{aligned}
$$

so that the IBVP governing the flow of the canonical Oldroyd-B fluid is given by (13)-(15).

The governing equations corresponding to anomalous Oldroyd- $\mathrm{B}$ fluids performing the same motion are obtained by replacing the inner time derivatives with left-sided Caputo fractional time derivatives $\partial_{t}^{\alpha}$ and $\partial_{t}^{\beta}$ defined in (1) for $0<\alpha \leq \beta<1$. Precisely, we entertain the following model:

$$
\begin{cases}\rho_{1}\left(1+\lambda_{1}^{\alpha} \frac{\partial^{\alpha}}{\partial t^{\alpha}}\right)\left[\frac{\partial u}{\partial t}\right]=\mu\left(1+\lambda_{2}^{\beta} \frac{\partial^{\beta}}{\partial t^{\beta}}\right)\left[\frac{\partial^{2} u}{\partial y^{2}}\right], & |y|<L, t>0, \\ u(L, t)=0, \quad u(-L, t)=A \frac{\mu^{2}}{\rho_{1}^{2} L^{4}} t^{2}, & t>0, \\ u(y, 0)=0=\frac{\partial u}{\partial t}(y, 0), & |y| \leq L .\end{cases}
$$

Note that the exponents $\alpha$ and $\beta$ on $\lambda_{1}$ and $\lambda_{2}$ are introduced in order to match the dimensions of different terms in (16). Furthermore, we can normalize the anomalous model 
(16) using the following dimensionless quantities:

$$
\hat{y}:=\frac{y}{L}, \quad \hat{u}:=\frac{u}{A}, \quad \hat{t}:=\frac{\mu}{\rho_{1} L^{2}} t, \quad \hat{\lambda}_{1}:=\lambda_{1} \frac{\mu}{\rho_{1} L^{2}}, \quad \hat{\lambda}_{2}:=\lambda_{2} \frac{\mu}{\rho_{1} L^{2}} .
$$

By (17), after dropping the hats for brevity and using the same notation for dimensionless quantities by abuse of notation, the IBVP (16) becomes

$$
\begin{cases}\left(1+\lambda_{1}^{\alpha} \frac{\partial^{\alpha}}{\partial t^{\alpha}}\right)\left[\frac{\partial u}{\partial t}\right]=\left(1+\lambda_{2}^{\beta} \frac{\partial^{\beta}}{\partial t^{\beta}}\right)\left[\frac{\partial^{2} u}{\partial y^{2}}\right], & |y|<1, t>0 \\ u(1, t)=0, \quad u(-1, t)=t^{2}, & t>0, \\ u(y, 0)=0=\frac{\partial u}{\partial t}(y, 0), & |y| \leq 1 .\end{cases}
$$

Remark 2.5 As a consequence of Remark 2.2, the anomalous Oldroyd-B model (16) reduces to the canonical Oldroyd-B model as $\alpha, \beta \rightarrow 1$. Moreover, (16) refers to a fractional Maxwell fluid as $\lambda_{2} \rightarrow 0$.

We end this section by introducing the function $\varphi(y, t)$ by

$$
\varphi(y, t):=u(y, t)+\frac{1}{2}(y-1) t^{2}
$$

Then, by invoking Proposition 2.3 we get

$$
\begin{cases}\left(1+\lambda_{1}^{\alpha} \frac{\partial^{\alpha}}{\partial t^{\alpha}}\right)\left[\frac{\partial \varphi}{\partial t}\right]-\left(1+\lambda_{2}^{\beta} \frac{\partial^{\beta}}{\partial t^{\beta}}\right)\left[\frac{\partial^{2} \varphi}{\partial y^{2}}\right] & \\ =[y-1]\left(t+B t^{1-\alpha}\right), & |y|<1, t>0, \\ \varphi(1, t)=0=\varphi(-1, t), & t>0, \\ \varphi(y, 0)=0=\frac{\partial \varphi}{\partial t}(y, 0), & |y| \leq 1,\end{cases}
$$

where the constant $B$ is defined by $B:=\frac{\lambda_{1}^{\alpha}}{\Gamma(2-\alpha)}$.

\section{Numerical approximation scheme}

The aim of this section is to discuss a numerical scheme for approximating velocity field satisfying the flow problem (18) over a finite interval of time $t \in[0, T]$ with some final control time $T>0$. Precisely, we consider

$$
\begin{cases}\left(1+\lambda_{1}^{\alpha} \frac{\partial^{\alpha}}{\partial t^{\alpha}}\right)\left[\frac{\partial \varphi}{\partial t}\right]-\left(1+\lambda_{2}^{\beta} \frac{\partial^{\beta}}{\partial t^{\beta}}\right)\left[\frac{\partial^{2} \varphi}{\partial y^{2}}\right] & \\ =(y-1)\left(t+B t^{1-\alpha}\right), & |y|<1, t \in(0, T), \\ \varphi(1, t)=0=\varphi(-1, t), & t \in(0, T), \\ \varphi(y, 0)=0=\frac{\partial \varphi}{\partial t}(y, 0), & |y| \leq 1 .\end{cases}
$$

A few useful notions and notation are collected below, and a discrete weak flow problem is derived in order to implement a standard Galerkin FEM blended with a so-called L1-finite difference approximation scheme following [41-43]. The well-posedness of the discrete and continuous problems can be proved using standard argument of Lax-Milgram in appropriate functional spaces; refer, for instance, to [34] for a special case of fractional Maxwell model. 


\subsection{Functional spaces and norms}

We denote the space of square-integrable functions over $\Omega=(-1,1)$ by $L^{2}(\Omega)$. Recall that $L^{2}(\Omega)$ is equipped with inner product and norm defined respectively by

$$
(\phi, \psi):=\int_{\Omega} \phi \psi d y \quad \text { and } \quad\|\phi\|_{0}:=\left(\int_{\Omega}|\phi|^{2} d y\right)^{1 / 2}, \quad \phi, \psi \in L^{2}(\Omega) .
$$

Henceforth, we fix the notation

$$
\begin{aligned}
& \langle\phi, \psi\rangle:=\left(\frac{\partial \phi}{\partial y}, \frac{\partial \psi}{\partial y}\right) \\
& \mathcal{L}_{t}^{\alpha}[\xi(\cdot)](t):=\left(1+\lambda_{1}^{\alpha} \frac{\partial^{\alpha}}{\partial t^{\alpha}}\right) \frac{\partial}{\partial t}[\xi(t)], \\
& \mathcal{Q}_{t}^{\beta}[\xi(\cdot)](t):=\left(1+\lambda_{2}^{\beta} \frac{\partial^{\beta}}{\partial t^{\beta}}\right)[\xi(t)] .
\end{aligned}
$$

Moreover, $H^{p}(\Omega)$ denotes the usual Sobolev space for $p>0$, and $H_{0}^{p}(\Omega)$ denotes the closure of $\mathcal{C}_{0}^{\infty}(\bar{\Omega})$ in $H^{p}(\Omega)$, where $\mathcal{C}_{0}^{\infty}(\bar{\Omega})$ represents the space of infinitely continuous functions having compact support in $\Omega$ (see, e.g., [47]). Recall that $H^{p}(\Omega)$ and $H_{0}^{p}(\Omega)$ are equipped with inner products and norms

$$
\begin{aligned}
& (\phi, \psi)_{H^{p}}:=(\phi, \psi)_{p}=\sum_{i=0}^{p}\left(\frac{d^{i} \phi}{d y^{i}}, \frac{d^{i} \psi}{d y^{i}}\right) \text { and }(\phi, \psi)_{H_{0}^{p}}:=(\phi, \psi)_{p, 0}=\left(\frac{d^{p} \phi}{d y^{p}}, \frac{d^{p} \psi}{d y^{p}}\right), \\
& \|\phi\|_{H^{p}}:=\|\phi\|_{p}=\left(\sum_{i=0}^{p}\left\|\frac{d^{i} \phi}{d y^{i}}\right\|_{0}^{2}\right)^{1 / 2} \text { and }\|\phi\|_{H_{0}^{p}}:=\|\phi\|_{p, 0}:=|\phi|_{H^{p}}=\left\|\frac{d^{p} \phi}{d y^{p}}\right\|_{0}
\end{aligned}
$$

where $|\cdot|$ represents a seminorm. Let us define the equivalent norm

$$
\|\phi\|_{1, *}:=\left(\left(1+\tau C_{\alpha}\right)\|\phi\|_{0}^{2}+\tau\left(1+C_{\beta}\right)\left\|\frac{\partial \phi}{\partial y}\right\|_{0}^{2}\right)^{1 / 2}
$$

for $H^{1}(\Omega)$, where $C_{\alpha}$ and $C_{\beta}$ are parameters depending on $\tau>0$ (a parameter to be made precise latter), material parameters $\lambda_{j}(j=1,2), \alpha$, and $\beta$ given by

$$
C_{\alpha}\left(\tau ; \lambda_{1}\right):=\lambda_{1}^{\alpha} \frac{\tau^{-(\alpha+1)}}{\Gamma(2-\alpha)} \quad \text { and } \quad C_{\beta}\left(\tau ; \lambda_{2}\right):=\lambda_{2}^{\beta} \frac{\tau^{-\beta}}{\Gamma(2-\beta)}
$$

It is easy to see that $\|\cdot\|_{1}$ and $\|\cdot\|_{1, *}$ are indeed equivalent norms on $H^{1}(\Omega)$ and subsequently on $H_{0}^{1}(\Omega)$.

Let $L^{2}(0, T ; V(\Omega))$ be the Hilbert space of functions $\phi$ from $[0, T]$ having values in a separable Hilbert space $V$ such that $\|\phi\|_{V} \in L^{2}(0, T)$ equipped with

$$
(\phi, \psi)_{L^{2}(0, T ; V(\Omega))}:=\int_{0}^{T}(\phi, \psi)_{V(\Omega)} d t \quad \text { and } \quad\|\phi\|_{L^{2}(0, T ; V(\Omega))}:=\sqrt{\int_{0}^{T}\|\phi\|_{V(\Omega)}^{2} d t} .
$$

Let us denote by $\mathcal{C}^{0}([0, T] ; V(\Omega))$ the space of continuous functions with norm

$$
\|\phi\|_{\mathcal{C}^{0}([0, T] ; V(\Omega))}:=\max _{t \in[0, T]}\|\phi\|_{V} .
$$


Analogously, for $n \in \mathbb{N}$,

$$
\mathcal{C}^{n}([0, T] ; V(\Omega)):=\left\{\phi \in \mathcal{C}^{0}([0, T] ; V(\Omega)) \mid \partial_{t}^{j} \phi \in \mathcal{C}^{0}([0, T] ; V(\Omega)), \forall j \leq n: j \in \mathbb{N}\right\}
$$

with norm

$$
\|\phi\|_{\mathcal{C}^{n}([0, T] ; V(\Omega))}:=\max _{j=0}^{n}\left(\left\|\partial_{t}^{j} \phi\right\|_{\mathcal{C}^{0}([0, T] ; V(\Omega))}\right)
$$

\subsection{Finite difference approximation}

For a fixed integer $m$, let $\tau:=T / m$ be the time step size, and let $t_{k}:=k \tau$ for $k=0,1,2, \ldots, m$. Consider the approximations

$$
\frac{\partial \varrho}{\partial t}(y, s) \simeq \frac{\partial \varrho}{\partial t}\left(y, t_{k}\right) \simeq \frac{\varrho\left(y, t_{k+1}\right)-\varrho\left(y, t_{k}\right)}{\tau}
$$

for all $t_{k} \leq s \leq t_{k+1}$ and

$$
\frac{\partial^{2} \varrho}{\partial t^{2}}(y, s) \simeq \frac{\partial^{2} \varrho}{\partial t^{2}}\left(y, t_{k}\right) \simeq \frac{\varrho\left(y, t_{k+1}\right)-2 \varrho\left(y, t_{k}\right)+\varrho\left(y, t_{k-1}\right)}{\tau^{2}}
$$

for all $t_{k-1} \leq s \leq t_{k+1}$ with $0<k<m$. Moreover, for $k=0$,

$$
\frac{\partial \varrho}{\partial t}\left(y, t_{0}\right) \simeq \frac{\varrho\left(y, t_{1}\right)-\varrho\left(y, t_{0}\right)}{\tau} \text { and } \frac{\partial^{2} \varrho}{\partial t^{2}}\left(y, t_{0}\right) \simeq \frac{1}{\tau}\left[\frac{\partial \varrho}{\partial t}\left(y, t_{1}\right)-\frac{\partial \varrho}{\partial t}\left(y, t_{0}\right)\right]
$$

Therefore, if $\varrho$ satisfies the initial conditions $\varrho(y, 0)=0=\partial \varrho / \partial t(y, 0)$, then

$$
\varrho\left(y, t_{1}\right) \simeq 0 \text { and } \frac{\partial^{2} \varrho}{\partial t^{2}}\left(y, t_{0}\right) \simeq \frac{1}{\tau} \frac{\partial \varrho}{\partial t}\left(x, t_{1}\right) \simeq \frac{1}{\tau^{2}}\left[\varrho\left(x, t_{1}\right)-\varrho\left(x, t_{0}\right)\right] \simeq 0
$$

Consequently, following Lin and $\mathrm{Xu}$ [41], the finite difference approximation to fractionalorder time derivative $\partial_{t}^{\beta}(0<\beta<1)$ for all $0 \leq k<m$ is given by

$$
\begin{aligned}
\frac{\partial^{\beta} \varrho}{\partial t^{\beta}}\left(y, t_{k+1}\right) & =\frac{1}{\Gamma(1-\beta)} \sum_{s=0}^{k} \int_{s \tau}^{(s+1) \tau} \frac{\partial \varrho(y, \xi)}{\partial \xi} \frac{d \xi}{\left(t_{k+1}-\xi\right)^{\beta}} \\
& \simeq \frac{1}{\Gamma(1-\beta)} \sum_{s=0}^{k} \frac{\varrho\left(y, t_{s+1}\right)-\varrho\left(y, t_{s}\right)}{\tau} \int_{s \tau}^{(s+1) \tau} \frac{d \xi}{\left(t_{k+1}-\xi\right)^{\beta}} \\
& =\frac{1}{\Gamma(1-\beta)} \sum_{p=0}^{k} \frac{\varrho\left(y, t_{k+1-p}\right)-\varrho\left(y, t_{k-p}\right)}{\tau} \int_{p \tau}^{(p+1) \tau} \frac{d \varepsilon}{\varepsilon^{\beta}} \\
& =\frac{\tau^{-\beta}}{\Gamma(2-\beta)}\left[\varrho\left(y, t_{k+1}\right)-\varrho\left(y, t_{k}\right)\right]+\frac{\tau^{-\beta}}{\Gamma(2-\beta)} \zeta_{k}^{\beta}[\varrho],
\end{aligned}
$$

where $b_{p}^{\beta}:=(p+1)^{1-\beta}-(p)^{1-\beta}, 0 \leq p \leq m$, and

$$
\zeta_{k}^{\beta}[\varrho]:=\sum_{s=1}^{k} b_{s}^{\beta}\left[\varrho\left(y, t_{k+1-s}\right)-\varrho\left(y, t_{k-s}\right)\right] \quad \text { with } \zeta_{0}^{\beta}[\varrho]:=0
$$


By (22) it can be observed immediately that $\zeta_{1}^{\gamma}[\varrho] \simeq 0$, for all $\gamma \in\{\alpha, \beta\}$. On the other hand, since $1<\alpha+1<2$, we have (see, e.g., [42]), for $0<k<m$,

$$
\begin{aligned}
& \frac{\partial^{\alpha+1} \varrho}{\partial t^{\alpha+1}}\left(y, t_{k+1}\right) \\
& \quad=\frac{1}{\Gamma(2-(\alpha+1))} \sum_{s=0}^{k} \int_{s \tau}^{(s+1) \tau} \frac{\partial^{2} \varrho(y, \xi)}{\partial \xi^{2}} \frac{d \xi}{\left(t_{k+1}-\xi\right)^{\alpha}} \\
& \quad=\frac{1}{\Gamma(1-\alpha)} \sum_{s=0}^{k} \int_{s \tau}^{(s+1) \tau} \frac{\partial^{2} \varrho\left(y, t_{s}\right)}{\partial \xi^{2}} \frac{d \xi}{\left(t_{k+1}-\xi\right)^{\alpha}} \\
& \quad=\frac{1}{\Gamma(1-\alpha)}\left[\int_{0}^{\tau} \frac{\partial^{2} \varrho\left(y, t_{0}\right)}{\partial \xi^{2}} \frac{d \xi}{\left(t_{k+1}-\xi\right)^{\alpha}}+\sum_{s=1}^{k} \int_{s \tau}^{(s+1) \tau} \frac{\partial^{2} \varrho\left(y, t_{s}\right)}{\partial \xi^{2}} \frac{d \xi}{\left(t_{k+1}-\xi\right)^{\alpha}}\right] \\
& \simeq \frac{1}{\Gamma(1-\alpha)} \sum_{s=1}^{k} \frac{\varrho\left(y, t_{s+1}\right)-2 \varrho\left(y, t_{s}\right)+\varrho\left(y, t_{s-1}\right)}{\tau^{2}} \int_{s \tau}^{(s+1) \tau} \frac{d \xi}{\left(t_{k+1}-\xi\right)^{\alpha}},
\end{aligned}
$$

where the last relation results from approximations (21) and (22). Changing the summation index, we obtain

$$
\begin{aligned}
\frac{\partial^{\alpha+1} \varrho}{\partial t^{\alpha+1}}\left(y, t_{k+1}\right)= & \frac{1}{\Gamma(1-\alpha)} \sum_{p=0}^{k-1} \frac{\varrho\left(y, t_{k+1-p}\right)-2 \varrho\left(y, t_{k-p}\right)+\varrho\left(y, t_{k-1-p}\right)}{\tau^{2}} \int_{p \tau}^{(p+1) \tau} \frac{d \varepsilon}{\varepsilon^{\alpha}} \\
= & \frac{\tau^{-\alpha-1}}{\Gamma(2-\alpha)}\left[\varrho\left(y, t_{k+1}\right)-2 \varrho\left(y, t_{k}\right)+\varrho\left(y, t_{k-1}\right)\right] \\
& +\frac{\tau^{-\alpha-1}}{\Gamma(2-\alpha)} \sum_{p=1}^{k-1} b_{p}^{\alpha}\left[\varrho\left(y, t_{k+1-p}\right)-2 \varrho\left(y, t_{k-p}\right)+\varrho\left(y, t_{k-1-p}\right)\right] .
\end{aligned}
$$

By the definition of memory terms $\zeta_{k-1}^{\alpha}[\varrho]$ and $\zeta_{k}^{\alpha}[\varrho]$ we get

$$
\begin{aligned}
\frac{\partial^{\alpha+1} \varrho}{\partial t^{\alpha+1}}\left(y, t_{k+1}\right)= & \frac{\tau^{-\alpha-1}}{\Gamma(2-\alpha)}\left[\varrho\left(y, t_{k+1}\right)-2 \varrho\left(y, t_{k}\right)+\varrho\left(y, t_{k-1}\right)\right] \\
& +\frac{\tau^{-\alpha-1}}{\Gamma(2-\alpha)}\left[\zeta_{k}^{\alpha}[\varrho]-\zeta_{k-1}^{\alpha}[\varrho]\right] .
\end{aligned}
$$

Therefore, $\mathcal{L}_{t}^{\alpha}[\varrho]$ and $\mathcal{Q}_{t}^{\beta}[\varrho]$ can be approximated for $0<k<m$ by

$$
\begin{aligned}
\mathcal{L}_{t}^{\alpha}[\varrho]\left(t_{k+1}\right)= & \left(\frac{\partial}{\partial t}+\lambda_{1}^{\alpha} \frac{\partial^{\alpha+1}}{\partial t^{\alpha+1}}\right)[\varrho]\left(t_{k+1}\right) \\
\simeq & \frac{1}{\tau}\left[\varrho\left(t_{k+1}\right)-\varrho\left(t_{k}\right)\right]+C_{\alpha}\left[\varrho\left(t_{k+1}\right)-2 \varrho\left(t_{k}\right)+\varrho\left(t_{k-1}\right)\right] \\
& +C_{\alpha}\left[\zeta_{k}^{\alpha}[\varrho]-\zeta_{k-1}^{\alpha}[\varrho]\right] \\
\mathcal{Q}_{t}^{\beta}[\varrho]\left(t_{k+1}\right)= & \left(1+\lambda_{2}^{\beta} \frac{\partial^{\beta}}{\partial t^{\beta}}\right)[\varrho]\left(t_{k+1}\right) \\
\simeq & \varrho\left(t_{k+1}\right)+C_{\beta}\left[\varrho\left(t_{k+1}\right)-\varrho\left(t_{k}\right)\right]+C_{\beta} \zeta_{k}^{\beta}[\varrho] .
\end{aligned}
$$


Finally, we conclude this section by defining the approximate time-discrete operators

$$
\begin{aligned}
& \widehat{\mathcal{L}}_{k+1}^{\alpha}[\varrho]:=\frac{\varrho_{k+1}-\varrho_{k}}{\tau}+C_{\alpha}\left[\varrho_{k+1}-2 \varrho_{k}+\varrho_{k-1}\right]+C_{\alpha}\left[\zeta_{k}^{\alpha}[\varrho]-\zeta_{k-1}^{\alpha}[\varrho]\right] \\
& \widehat{\mathcal{Q}}_{k+1}^{\beta}[\varrho]:=\varrho_{k+1}+C_{\beta}\left[\varrho_{k+1}-\varrho_{k}\right]+C_{\beta} \zeta_{k}^{\beta}[\varrho]
\end{aligned}
$$

such that

$$
\mathcal{L}_{t}^{\alpha}[\varrho]\left(t_{k+1}\right)=\widehat{\mathcal{L}}_{k+1}^{\alpha}[\varrho]+R_{\alpha, 1}^{k+1}+O(\tau) \quad \text { and } \quad \mathcal{Q}_{t}^{\beta}[\varrho]\left(t_{k+1}\right)=\widehat{\mathcal{L}}_{k+1}^{\beta}[\varrho]+R_{\beta, 2}^{k+1}
$$

where $\varrho_{k}$ is the approximation to $\varrho\left(t_{k}\right)$, and $R_{\alpha, 1}^{k+1}$ and $R_{\beta, 2}^{k+1}$ are the truncation error terms. We provide further discussion on the truncation error to Section 4.1 and present a spatial discretization scheme in the next section.

\subsection{Galerkin finite element approximation}

Let $-1=y_{1}<y_{2}<\cdots<y_{n}<y_{n+1}=1$. Define the partition of the domain $\Omega$ into $n$ subdomains $\Omega_{i}=\left(y_{i}, y_{i+1}\right)$ for $i=1,2, \ldots, n$ such that

$$
\bar{\Omega}=\bigcup_{i=1}^{n} \bar{\Omega}_{i} \quad \text { and } \quad \Omega_{i} \bigcap \Omega_{j}=\emptyset, \quad \forall i \neq j .
$$

Let $h$ be the uniform length of elements $\Omega_{i}$, that is, $h:=2 / n:=y_{i+1}-y_{i}$. Define the sequence of finite-dimensional approximation subspaces $\left\{V_{0}^{h}(\Omega)\right\}_{h>0}$ of $H_{0}^{1}(\Omega)$ by

$$
V_{0}^{h}(\Omega):=\left\{\phi \in H_{0}^{1}(\Omega) \mid \phi_{\left.\right|_{\Omega_{i}}} \in \wp_{r}\left(\Omega_{i}\right), \forall i=1,2, \ldots, n\right\},
$$

where $\wp_{r}\left(\Omega_{i}\right)$ is a Lagrange interpolation space of polynomials with degree at most $r$ over the element $\Omega_{i}$ for each $i=1,2, \ldots, n$.

Consider the following weak formulation of the flow problem (19).

Weak Form Find $\varphi \in \mathcal{C}^{1}\left([0, T] ; H_{0}^{1}(\Omega)\right)$ such that

$$
\left\{\begin{array}{l}
\mathcal{L}_{t}^{\alpha}(\varphi(y, t), \chi)+\mathcal{Q}_{t}^{\beta}\langle\varphi(y, t), \chi\rangle=\left((y-1)\left(t+B t^{1-\alpha}\right), \chi\right) \\
\varphi(y, 0)=0=\frac{\partial \varphi}{\partial t}(y, 0)
\end{array}\right.
$$

for all $\chi \in H_{0}^{1}(\Omega)$.

In the sequel, we derive a space and time discrete weak formulation of the problem (19) using (28). Let $\varphi_{h}$ be an approximate solution to $(28)$ in $\mathcal{C}^{1}\left([0, T] ; V_{0}^{h}\right)$, that is, the solution to following problem.

Semidiscrete weak form Find $\varphi_{h} \in \mathcal{C}^{1}\left([0, T] ; V_{0}^{h}(\Omega)\right)$ such that

$$
\left\{\begin{array}{l}
\mathcal{L}_{t}^{\alpha}\left(\varphi_{h}(y, t), \chi_{h}\right)+\mathcal{Q}_{t}^{\beta}\left\langle\varphi_{h}(y, t), \chi_{h}\right\rangle=\left((y-1)\left(t+B t^{1-\alpha}\right), \chi_{h}\right) \\
\varphi_{h}(y, 0)=0=\frac{\partial \varphi_{h}}{\partial t}(y, 0)
\end{array}\right.
$$

for all $\chi_{h} \in V_{0}^{h}(\Omega)$. 
Using approximations (24)-(26) in (29) for a fixed time $t_{k+1}(0<k<m)$, the discrete weak form of the flow problem (19) is given by

$$
\left\{\begin{array}{l}
\text { Find } \varphi_{h}\left(\cdot, t_{k+1}\right) \in V_{0}^{h}(\Omega) \text { such that } \forall \chi_{h} \in V_{0}^{h}(\Omega): \\
\quad \widehat{\mathcal{L}}_{k+1}^{\alpha}\left(\varphi_{h}\left(y, t_{k+1}\right), \chi_{h}\right)+\widehat{\mathcal{Q}}_{k+1}^{\beta}\left\langle\varphi_{h}\left(y, t_{k+1}\right), \chi_{h}\right\rangle+R_{\alpha, 1}^{k+1}(y)+R_{\beta, 2}^{k+1}(y) \\
\quad=\left((y-1)\left(t_{k+1}+B t_{k+1}^{1-\alpha}\right), \chi_{h}\right), \\
\varphi_{h}^{0}(y)=0=\varphi_{h}^{1}(y),
\end{array}\right.
$$

where $\varphi_{h}^{0}(\cdot)=\varphi_{h}\left(\cdot, t_{0}\right)$ and $\varphi_{h}^{1}(\cdot)=\varphi_{h}\left(\cdot, t_{1}\right)$. On the other hand, recall that the approximate solution $\varphi_{h}$ to (29) can be written as

$$
\varphi_{h}(y, t)=\sum_{p=1}^{N_{h}} \varphi_{p}(t) W_{h}^{p}(y), \quad y \in \bar{\Omega},
$$

where $\left\{W_{h}^{p} \mid p=1,2, \ldots, N_{h}\right\}$ forms a basis of $V_{0}^{h}(\Omega)$ with $N_{h}:=\operatorname{dim}\left(V_{0}^{h}\right)$, and $\varphi_{p}$ are the values to be determined. Therefore, choosing $\chi_{h}$ as $W_{h}^{q}$ for different values of $q=1,2, \ldots, N_{h}$ finally leads to the following system of equations:

$$
\left\{\begin{array}{l}
\mathbb{A}^{h} \widehat{\mathcal{L}}_{k+1}^{\alpha}\left[\Phi_{h}\right]\left(t_{k+1}\right)+\mathbb{B}^{h} \widehat{\mathcal{Q}}_{k+1}^{\beta}\left[\Phi_{h}\right]\left(t_{k+1}\right)+\mathbf{E}^{k+1}\left(\mathbf{W}_{h}\right)=\mathbf{G}_{h}^{k+1}\left(\mathbf{W}_{h}\right), \\
\Phi_{h}^{0}=\mathbf{0}=\Phi_{h}^{1},
\end{array}\right.
$$

where, for all $p, q=1,2, \ldots, N_{h}$,

$$
\left\{\begin{array}{l}
\left(\Phi_{h}\right)_{p}:=\varphi_{p}, \\
\left(\mathbf{W}_{h}\right)_{p}:=W_{h}^{p}, \\
\left(\mathbb{A}^{h}\right)_{q p}:=\left(W_{h}^{p}, W_{h}^{q}\right), \\
\left(\mathbb{B}^{h}\right)_{q p}:=\left\langle W_{h}^{p}, W_{h}^{q}\right\rangle, \\
\left(\mathbf{G}_{h}^{k+1}\left(\mathbf{W}_{h}\right)\right)_{p}:=\left((y-1)\left(t_{k+1}+B t_{k+1}^{1-\alpha}\right), W_{h}^{p}\right), \\
\left(\mathbf{E}^{k+1}\left(\mathbf{W}_{h}\right)\right)_{p}:=\left(R_{\alpha, 1}^{k+1}+R_{\beta, 2}^{k+1}, W_{h}^{p}\right) .
\end{array}\right.
$$

The finite difference-finite element approximation scheme for the flow problem (19) can be described in two steps. In the sequel, the approximation to $\Phi_{h}\left(t_{k+1}\right)$ is denoted by $\Phi_{h}^{k+1}$. In order to initiate the iterative scheme, the first two terms $\Phi_{h}^{0}$ and $\Phi_{h}^{1}$ are required. Note that, by initial conditions,

$$
\Phi_{h}^{0}=\Phi_{h}\left(t_{0}\right)=\mathbf{0} \quad \text { and } \quad \Phi_{h}^{1}=\Phi_{h}\left(t_{1}\right)=\mathbf{0} .
$$

For $1<k<m$, the approximate solution $\Phi_{h}^{k+1}$ can be obtained by successively solving

$$
\mathbb{A}^{h} \widehat{\mathcal{L}}_{k+1}^{\alpha}\left[\Phi_{h}\right]\left(t_{k+1}\right)+\mathbb{B}^{h} \widehat{\mathcal{Q}}_{k+1}^{\beta}\left[\Phi_{h}\right]\left(t_{k+1}\right)=\mathbf{G}_{h}^{k+1}\left(\mathbf{W}_{h}\right) .
$$

\section{Analysis of numerical scheme}

This section is dedicated to the stability and error analysis of the numerical scheme established in Section 3. In the sequel, $C$ represents a generic constant independent of $\tau$ and $h$ but dependent on $\varphi, \alpha, \beta, \lambda_{1}, \lambda_{2}, u$, and $T$ and may differ from step to step. 


\subsection{Truncation error}

We recall that the truncation errors for the finite difference approximations of time fractional Caputo derivatives of order $\alpha+1$ and $\beta$ are given respectively by

$$
\begin{aligned}
R_{\alpha, 1}^{k+1}= & \frac{\partial^{\alpha+1} \varrho}{\partial t^{\alpha+1}}\left(t_{k+1}\right)-\frac{\tau^{-\alpha-1}}{\Gamma(2-\alpha)}\left[\varrho\left(t_{k+1}\right)-2 \varrho\left(t_{k}\right)+\varrho\left(t_{k-1}\right)\right] \\
& -\frac{\tau^{-\alpha-1}}{\Gamma(2-\alpha)}\left[\zeta_{k}^{\alpha}[\varrho]-\zeta_{k-1}^{\alpha}[\varrho]\right] \\
= & \frac{1}{\Gamma(1-\alpha)} \sum_{s=0}^{k} \int_{t_{s}}^{t_{s+1}}\left[\frac{\partial^{2} \varrho(\xi)}{\partial \xi^{2}}-\frac{\partial^{2} \varrho\left(t_{s}\right)}{\partial t^{2}}-\frac{\tau^{2}}{12} \frac{\partial^{4} \varrho\left(t_{s}\right)}{\partial \xi^{4}}\right. \\
& \left.+O\left(\tau^{4}\right)\right] \frac{d \xi}{\left(t_{k+1}-\xi\right)^{\alpha}}, \\
R_{\beta, 2}^{k+1}= & \frac{\partial^{\beta} \varrho}{\partial t^{\beta}}\left(t_{k+1}\right)-\frac{\tau^{-\beta}}{\Gamma(2-\beta)}\left[\varrho\left(t_{k+1}\right)-\varrho\left(t_{k}\right)\right]-\frac{\tau^{-\beta}}{\Gamma(2-\beta)} \zeta_{k}^{\beta}[\varrho] \\
= & \frac{1}{\Gamma(1-\beta)} \sum_{s=0}^{k} \int_{t_{s}}^{t_{s+1}}\left[\frac{\partial \varrho(\xi)}{\partial \xi}-\frac{\partial \varrho\left(t_{s}\right)}{\partial t}-\frac{\tau}{2} \frac{\partial^{2} \varrho\left(t_{s}\right)}{\partial \xi^{2}}\right. \\
& \left.+O\left(\tau^{2}\right)\right] \frac{d \xi}{\left(t_{k+1}-\xi\right)^{\beta}} .
\end{aligned}
$$

The following truncation error bounds hold. We refer the interested reader to $[41,42]$ for further details.

Lemma 4.1 If $\varrho \in \mathcal{C}^{4}([0, T])$, then, for all $1<k<m$,

$$
\begin{aligned}
& \left|R_{\alpha, 1}^{k+1}\right| \leq C \max _{t \in[0, T]}\left|\frac{\partial^{3} \varrho(t)}{\partial t^{3}}\right| \frac{k^{1-\alpha}}{\Gamma(2-\alpha)} \tau^{2-\alpha}, \\
& \left|R_{\beta, 2}^{k+1}\right| \leq C \max _{t \in[0, T]}\left|\frac{\partial^{2} \varrho(t)}{\partial t^{2}}\right| \frac{k^{1-\beta}}{\Gamma(2-\beta)} \tau^{2-\beta} .
\end{aligned}
$$

As an immediate consequence of Lemma 4.1, the following result is evident.

Lemma 4.2 (Truncation error) If $\varrho \in \mathcal{C}^{4}([0, T])$, then, for all $1<k<m$,

$$
\begin{aligned}
& \left|\mathcal{L}_{t}^{\alpha}[\varrho]\left(t_{k+1}\right)-\widehat{\mathcal{L}}_{k+1}^{\alpha}[\varrho]\right| \leq C \tau+\left|R_{\alpha, 1}^{k+1}\right| \leq C\left(\tau+\tau^{2-\alpha}\right), \\
& \left|\mathcal{Q}_{t}^{\beta}[\varrho]\left(t_{k+1}\right)-\widehat{\mathcal{Q}}_{k+1}^{\beta}[\varrho]\right| \leq\left|R_{\beta, 2}^{k+1}\right| \leq C\left(\tau^{2-\beta}\right) .
\end{aligned}
$$

\subsection{Stability of discrete problem}

This section is dedicated to proving the stability of the discrete weak problem

$$
\left\{\begin{array}{c}
\text { Find } \varphi_{h}^{k+1} \in V_{0}^{h}(\Omega) \text { such that } \forall \chi_{h} \in V_{0}^{h}(\Omega) \text { : } \\
\left.\quad+\tau C_{\alpha}\right)\left(\varphi_{h}^{k+1}, \chi_{h}\right)+\tau\left(1+C_{\beta}\right)\left\langle\varphi_{h}^{k+1}, \chi_{h}\right\rangle \\
\quad=\mathcal{M}_{k}\left[\varphi_{h}^{k}, \chi_{h}\right]+\tau\left(g(y)\left(t_{k+1}+B t_{k+1}^{1-\alpha}\right), \chi_{h}\right), \\
\varphi_{h}^{0}=0=\varphi_{h}^{1}, \quad 0<k<m,
\end{array}\right.
$$


where $\varphi_{h}^{k+1}$ is the approximation of $\varphi_{h}\left(\cdot, t_{k+1}\right)$, and

$$
\begin{aligned}
g(y):=(y-1), & \\
\mathcal{M}_{k}\left[\varphi_{h}^{k}, \chi_{h}\right]:= & \left(1+2 \tau C_{\alpha}\right)\left(\varphi_{h}^{k}, \chi_{h}\right)+\tau C_{\beta}\left\langle\varphi_{h}^{k}, \chi_{h}\right\rangle-\tau C_{\alpha}\left(\varphi_{h}^{k-1}, \chi_{h}\right) \\
& -\tau C_{\alpha}\left(\zeta_{k}^{\alpha}\left[\varphi_{h}\right]-\zeta_{k-1}^{\alpha}\left[\varphi_{h}\right], \chi_{h}\right)-\tau C_{\beta}\left\langle\zeta_{k}^{\beta}\left[\varphi_{h}\right], \chi_{h}\right\rangle .
\end{aligned}
$$

The following stability result holds.

Theorem 4.3 The discrete problem (32) is unconditionally stable in the sense that, for $\tau>0$ and for all $j$ such that $2 \leq j \leq m$,

$$
\left\|\varphi_{h}^{j}\right\|_{1, *} \leq C\|g\|_{0} .
$$

Proof Remark that $\varphi_{h}^{0}=\varphi_{h}^{1}=0$, and thus

$$
\left\|\varphi_{h}^{0}\right\|_{1, *}=\left\|\varphi_{h}^{1}\right\|_{1, *}=0 \leq\|g\|_{0} .
$$

In order to prove the stability estimate (33) for $2 \leq j \leq m$, we use mathematical induction.

1. Initial step: $(j=2)$. Taking $\chi_{h}=\varphi_{h}^{2}$ and $k=1$ in (32) yields

$$
\left\|\varphi_{h}^{2}\right\|_{1, *}^{2} \leq \mathcal{M}_{1}\left[\varphi_{h}^{1}, \varphi_{h}^{2}\right]+C\|g\|_{0}\left\|\varphi_{h}^{2}\right\|_{0}
$$

By initial conditions and the definition of operator $\mathcal{M}_{1}$ we obtain

$$
\mathcal{M}_{1}\left[\varphi_{h}^{1}, \varphi_{h}^{2}\right]=\left(1+2 \tau C_{\alpha}\right)\left(\varphi_{h}^{1}, \varphi_{h}^{2}\right)+\tau C_{\beta}\left\langle\varphi_{h}^{1}, \varphi_{h}^{2}\right\rangle+\tau C_{\alpha}\left(\varphi_{h}^{0}, \varphi_{h}^{2}\right)=0 .
$$

Consequently, inequality (34), together with the inequality $\left\|\varphi_{h}^{2}\right\|_{0} \leq\left\|\varphi_{h}^{2}\right\|_{1}$, yields

$$
\left\|\varphi_{h}^{2}\right\|_{1, *} \leq C\|g\|_{0}
$$

2. Supposition step: $(j<m)$. Assume that estimate (33) holds for $2<j<m$, that is, there exists a constant $C$ independent on $\tau$ and $h$ such that $\left\|\varphi_{h}^{j}\right\|_{1, *} \leq C\|g\|_{0}$.

3. Induction step: $(j=m)$. It is evident from the definition of $b_{k}^{\gamma}$ that

$$
1=b_{0}^{\gamma}>b_{1}^{\gamma}>\cdots>b_{k}^{\gamma} \rightarrow 0 \quad \text { as } k \rightarrow+\infty
$$

Taking $\chi_{h}=\varphi_{h}^{m}$ and $k=m-1$ in (32) yields

$$
\left\|\varphi_{h}^{m}\right\|_{1, *}^{2} \leq \mathcal{M}_{m-1}\left[\varphi_{h}^{m-1}, \varphi_{h}^{m}\right]+C\|g\|_{0}\left\|\varphi_{h}^{m}\right\|_{0} .
$$

Again, by the definition of the operator $\mathcal{M}_{m-1}$ we have

$$
\begin{aligned}
\mathcal{M}_{m-1} & {\left[\varphi_{h}^{m-1}, \varphi_{h}^{m}\right] } \\
= & \left(1+2 \tau C_{\alpha}\right)\left(\varphi_{h}^{m-1}, \varphi_{h}^{m}\right)+\tau C_{\beta}\left\langle\varphi_{h}^{m-1}, \varphi_{h}^{m}\right\rangle+\tau C_{\alpha}\left(\varphi_{h}^{m-2}, \varphi_{h}^{m}\right) \\
& +\tau C_{\alpha}\left(\zeta_{m-1}^{\alpha}\left[\varphi_{h}\right]-\zeta_{m-2}^{\alpha}\left[\varphi_{h}\right], \varphi_{h}^{m}\right)+\tau C_{\beta}\left\langle\zeta_{m-1}^{\beta}\left[\varphi_{h}\right], \varphi_{h}^{m}\right\rangle
\end{aligned}
$$




$$
\begin{aligned}
\leq & \left(1+2 \tau C_{\alpha}\right)\left\|\varphi_{h}^{m-1}\right\|_{0}\left\|\varphi_{h}^{m}\right\|_{0}+\tau C_{\beta}\left\|\frac{\partial \varphi_{h}^{m-1}}{\partial y}\right\|_{0}\left\|\frac{\partial \varphi_{h}^{m}}{\partial y}\right\|_{0} \\
& +\tau C_{\alpha}\left\|\varphi_{h}^{m-2}\right\|_{0}\left\|\varphi_{h}^{m}\right\|_{0}+\tau C_{\beta}\left\|\frac{\partial \zeta_{m-1}^{\beta}\left[\varphi_{h}\right]}{\partial y}\right\|_{0}\left\|\frac{\partial \varphi_{h}^{m}}{\partial y}\right\|_{0} \\
& +\tau C_{\alpha}\left\|\zeta_{m-1}^{\alpha}\left[\varphi_{h}\right]\right\|_{0}\left\|\varphi_{h}^{m}\right\|_{0}+\tau C_{\alpha}\left\|\zeta_{m-2}^{\alpha}\left[\varphi_{h}\right]\right\|_{0}\left\|\varphi_{h}^{m}\right\|_{0} \\
\leq & C \max \left(1+2 \lambda_{1}^{\alpha} \frac{T^{-\alpha}}{\Gamma(2-\alpha)}, \lambda_{2}^{\beta} \frac{T^{1-\beta}}{\Gamma(2-\beta)}\right)\|g\|_{0}\left\|\varphi_{h}^{m}\right\|_{1} .
\end{aligned}
$$

To get the last inequality, we have used the assumption step and the fact that

$$
\begin{aligned}
\left\|\zeta_{p}^{\gamma}[\varphi]\right\|_{1, *} & =\left\|\sum_{s=1}^{p} b_{s}^{\gamma}\left(\varphi_{h}^{p+1-s}-\varphi_{h}^{p-s}\right)\right\|_{1, *} \\
& \leq \sum_{s=1}^{p}\left(\left\|\varphi_{h}^{p+1-s}\right\|_{1, *}+\left\|\varphi_{h}^{p-s}\right\|_{1, *}\right) \leq C\|g\|_{0}
\end{aligned}
$$

for all $p<m$, which holds again by the assumption step and relation (35). This completes the proof together with (36).

\subsection{Convergence of numerical scheme}

Let $\pi_{h}: H_{0}^{1}(\Omega) \rightarrow V_{0}^{h}(\Omega)$ be the interpolation operator from $H_{0}^{1}(\Omega)$ into $V_{0}^{h}(\Omega)$ defined by

$$
\left\langle\pi_{h} \phi, v_{h}\right\rangle=\left\langle\phi_{h}, v_{h}\right\rangle, \quad \forall v_{h} \in V_{0}^{h}(\Omega), \phi_{h} \in V_{0}^{h}, \phi \in H_{0}^{1}(\Omega) .
$$

Let us define the error term $r_{\alpha}^{k+1}$ by

$$
r_{\alpha}^{k+1}(y):=\mathcal{L}_{t}^{\alpha}\left[\varphi_{h}\left(y, t_{k+1}\right)\right]-\widehat{\mathcal{L}}_{k+1}^{\alpha}\left[\pi_{h} \varphi\left(y, t_{k+1}\right)\right]
$$

Then, we have the following error bounds.

Lemma 4.4 There exists a constant $C>0$ depending on $\alpha, \lambda_{1}, \varphi$, and $T$ such that

$$
\left\|r_{\alpha}^{k+1}\right\|_{1} \leq C\left(h^{r+1}+\tau^{2-\alpha}\right)
$$

Proof Note that

$$
\begin{aligned}
\mathcal{L}_{t}^{\alpha} & {\left[\varphi_{h}\left(y, t_{k+1}\right)\right]-\widehat{\mathcal{L}}_{k+1}^{\alpha}\left[\pi_{h} \varphi\left(y, t_{k+1}\right)\right] } \\
& =\left(1-\pi_{h}\right) \mathcal{L}_{t}^{\alpha}\left[\varphi_{h}\left(y, t_{k+1}\right)\right]+\pi_{h}\left[\mathcal{L}_{t}^{\alpha}\left[\varphi_{h}\left(y, t_{k+1}\right)\right]-\widehat{\mathcal{L}}_{k+1}^{\alpha}\left[\varphi_{h}\left(y, t_{k+1}\right)\right]\right] .
\end{aligned}
$$

Therefore, recalling the truncation error estimates from Lemma 4.2 and the interpolation error estimates from $[48,49]$, we conclude that estimate (37) holds.

Remark from the discrete weak formulation (30) that, for $\chi_{h} \in V_{0}^{h}(\Omega) \subset H_{0}^{1}(\Omega)$ and a fixed $t_{k+1}$,

$$
\begin{aligned}
& \widehat{\mathcal{L}}_{k+1}^{\alpha}\left(\pi_{h} \varphi\left(y, t_{k+1}\right), \chi_{h}\right)+\widehat{\mathcal{Q}}_{k+1}^{\beta}\left\langle\pi_{h} \varphi\left(y, t_{k+1}\right), \chi_{h}\right\rangle+\left(R_{h}^{k+1}(y), \chi_{h}\right) \\
& \quad=\left(g(y)\left(t_{k+1}+B t_{k+1}^{1-\alpha}\right), \chi_{h}\right),
\end{aligned}
$$


where

$$
R_{h}^{k+1}(y):=R_{\alpha, 1}^{k+1}(y)+R_{\beta, 2}^{k+1}(y)+r_{\alpha}^{k+1}(y)
$$

The main result of this section is the following:

Theorem 4.5 (Convergence) Let $\varphi(\cdot, t)$ and $\varphi_{h}^{k+1}(\cdot)$ be the solutions to (19) and (32), respectively. Then there exists a constant $C>0$ independent on $h$ and $\tau$ such that

$$
\left\|\varphi\left(\cdot, t_{k+1}\right)-\varphi_{h}^{k+1}(\cdot)\right\|_{1, *} \leq C\left(h^{r+1}+\tau^{2}\right), \quad 0 \leq k<m .
$$

Proof The convergence estimate (39) can be proved by arguments analogous to those in the proof of [41], Theorem 3.2, and that of [43], Theorem 2.1. The key ingredients of the proof are further presented for completeness.

We split the error term $\left\|\varphi\left(\cdot, t_{k+1}\right)-\varphi_{h}^{k+1}(\cdot)\right\|_{1, *}$ as

$$
\begin{aligned}
\left\|\varphi\left(\cdot, t_{k+1}\right)-\varphi_{h}^{k+1}(\cdot)\right\|_{1, *} \leq & \left\|\varphi\left(\cdot, t_{k+1}\right)-\pi_{h} \varphi\left(\cdot, t_{k+1}\right)\right\|_{1, *} \\
& +\left\|\pi_{h} \varphi\left(\cdot, t_{k+1}\right)-\varphi_{h}^{k+1}(\cdot)\right\|_{1, *}
\end{aligned}
$$

Then the first term on the right-hand side (RHS) is well understood [48, 49], and we have

$$
\left\|\varphi\left(\cdot, t_{k+1}\right)-\pi_{h} \varphi\left(\cdot, t_{k+1}\right)\right\|_{1, *} \leq C h^{r+1} .
$$

In order to estimate the second term on the RHS, let

$$
\epsilon_{h}^{k+1}(\cdot):=\varphi_{h}^{k+1}(\cdot)-\pi_{h} \varphi\left(\cdot, t_{k+1}\right) .
$$

Then, by (38) and (32),

$$
\widehat{\mathcal{L}}_{k+1}^{\alpha}\left(\epsilon_{h}^{k+1}, \chi_{h}\right)+\widehat{\mathcal{Q}}_{k+1}^{\beta}\left\langle\epsilon_{h}^{k+1}, \chi_{h}\right\rangle=\left(R_{h}^{k+1}, \chi_{h}\right), \quad \forall \chi_{h} \in V_{0}^{h}(\Omega) .
$$

Choosing $\chi_{h}:=\epsilon_{h}^{k+1}$ in this equation, we arrive at

$$
\widehat{\mathcal{L}}_{k+1}^{\alpha}\left(\epsilon_{h}^{k+1}, \epsilon_{h}^{k+1}\right)+\widehat{\mathcal{Q}}_{k+1}^{\beta}\left\langle\epsilon_{h}^{k+1}, \epsilon_{h}^{k+1}\right\rangle=\left(R_{h}^{k+1}, \epsilon_{h}^{k+1}\right)
$$

or, equivalently,

$$
\left\|\epsilon_{h}^{k+1}\right\|_{1, *} \leq \mathcal{M}_{k}\left[\epsilon_{h}^{k}, \epsilon_{h}^{k+1}\right]+C \tau\left|R_{h}^{k+1}\right|\left\|\epsilon_{h}^{k+1}\right\|_{1, *}
$$

By Lemmas 4.1 and 4.4 the second term on the RHS of (41) can be controlled by $O\left(h^{r+1}+\right.$ $\tau^{2}$ ). In order to control the first term on the RHS of (41), we use the arguments analogous to those in Theorem 4.3 and prove that

$$
\mathcal{M}_{k}\left[\epsilon_{h}^{k}, \epsilon_{h}^{k+1}\right] \leq C\left(\left\|\epsilon_{h}^{k}\right\|_{1, *}+\left\|\epsilon_{h}^{k-1}\right\|_{1, *}\right)\left\|\epsilon_{h}^{k}\right\|_{1, *}
$$


Then using mathematical induction together with the fact that $\epsilon_{h}^{0}=\epsilon_{h}^{1}=0$, it can be proved in a similar fashion as in Theorem 4.3 that

$$
\left\|\epsilon_{h}^{k+1}\right\|_{1, *} \leq C\left(h^{r+1}+\tau^{2}\right)
$$

which consequently leads to the conclusion together with (40).

\section{Numerical results and discussion}

In this section, we present some numerical results showing the validity of the approximation scheme and discuss the approximate velocity profile.

\subsection{Validation of numerical scheme}

In order to validate the numerical scheme, we fabricate an exact solution and compare it with the approximated solution obtained using the numerical scheme established in Section 3 with $\wp_{1}$-elements. In order to construct an exact solution of the model, we introduce an artificial source term $F_{\text {art }}$ on the RHS of the first equation of (19) to arrive at

$$
\left(1+\lambda_{1}^{\alpha} \frac{\partial^{\alpha}}{\partial t^{\alpha}}\right)\left[\frac{\partial \varphi}{\partial t}\right]-\left(1+\lambda_{2}^{\beta} \frac{\partial^{\beta}}{\partial t^{\beta}}\right)\left[\frac{\partial^{2} \varphi}{\partial y^{2}}\right]=(y-1)\left(t+B t^{1-\alpha}\right)+F_{\text {art }}(y, t) .
$$

Then by choosing any smooth function $\varphi_{\mathrm{ex}}$ that satisfies both initial and boundary conditions of the model problem (19) we can easily find the corresponding source term $F_{\text {art }}$ by inserting $\varphi_{\mathrm{ex}}$ in (43). The function $\varphi_{\mathrm{ex}}$ then becomes an exact solution of equation (43) subject to initial and boundary conditions as in (19). Toward this end, we choose $\varphi_{\mathrm{ex}}(y, t)=t^{2} \sin \pi y$ in this subsection. In fact, the artificial source term in (43) is considered only to validate the numerical scheme and to discuss its convergence. For the numerical simulations of the model problem (19) and for discussing the velocity profile, we use the original source term and the corresponding numerical solution.

In Figure 2, the transient profiles of the exact (fabricated) and approximated solutions are compared over the time interval $[0,1]$. The results show a very good match between

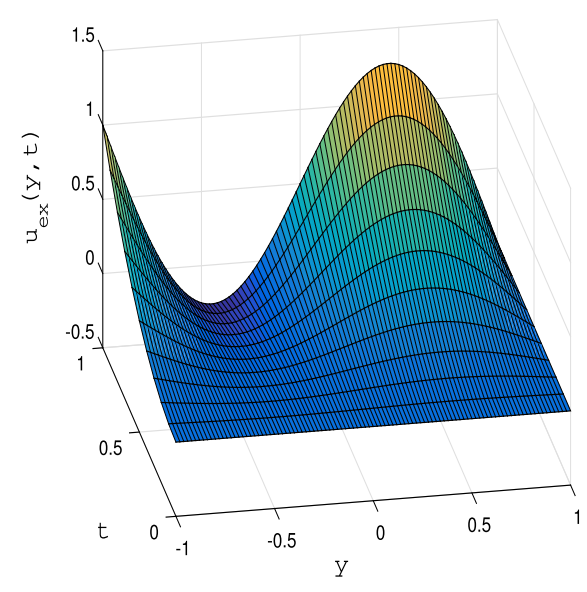

(a) Exact solution.

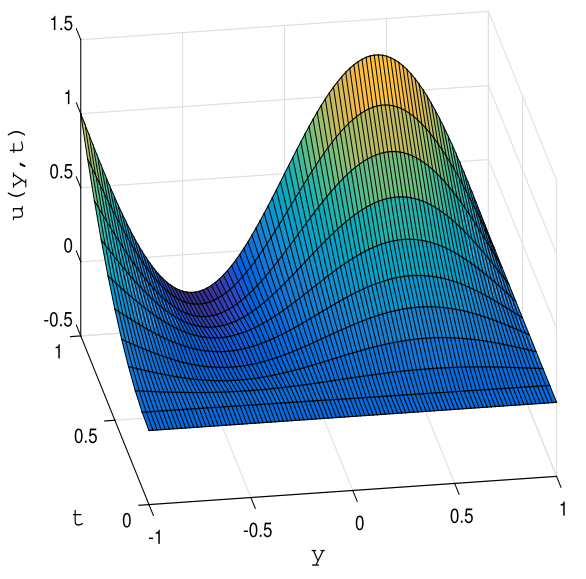

(b) Approximate solution.

Figure 2 Comparison of exact and approximate solutions over $(y, t) \in[-1,1] \times[0,1]$. 


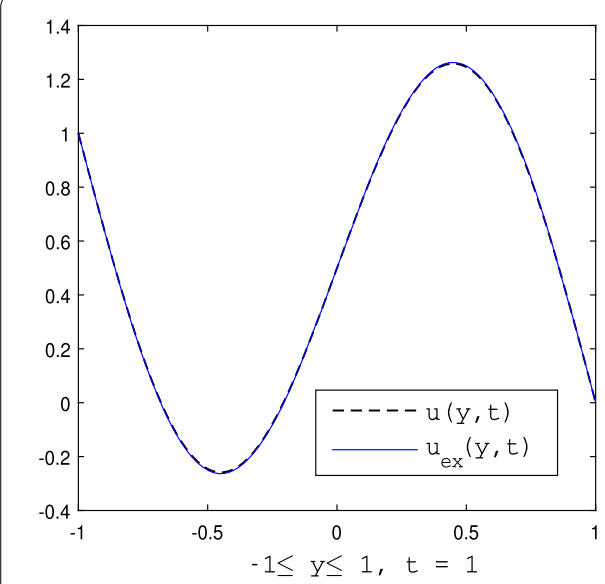

(a) Solution curves at $t=1$.

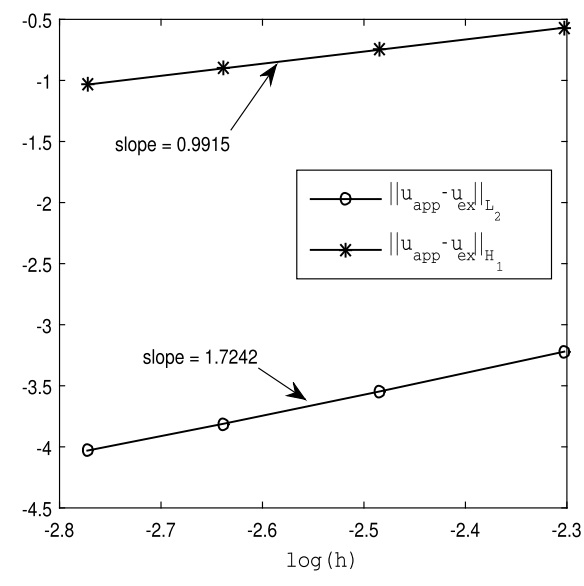

(b) Error in log scale for $h=10,20,30,40$.

Figure 3 Numerical error estimation.

Table 1 Convergence rates in $L^{2}$ and $H^{1}$ norms

\begin{tabular}{lll}
\hline $\begin{array}{l}\text { Number of } \\
\text { elements }\end{array}$ & $\left\|u-u_{\text {ex }}\right\|_{\mathbf{L}^{\mathbf{2}}(\boldsymbol{\Omega})}$ & $\left\|\boldsymbol{u}-\boldsymbol{u}_{\mathbf{e x}}\right\|_{\boldsymbol{H}^{\mathbf{1}}(\boldsymbol{\Omega})}$ \\
\hline 60 & $5.0077 \times 10^{-3}$ & $9.5930 \times 10^{-2}$ \\
70 & $4.7797 \times 10^{-3}$ & $8.2534 \times 10^{-2}$ \\
80 & $4.6342 \times 10^{-3}$ & $7.2526 \times 10^{-2}$ \\
90 & $4.5357 \times 10^{-3}$ & $6.4777 \times 10^{-2}$ \\
100 & $4.4659 \times 10^{-3}$ & $5.8608 \times 10^{-2}$ \\
110 & $4.4146 \times 10^{-3}$ & $5.3588 \times 10^{-2}$ \\
120 & $4.3758 \times 10^{-3}$ & $4.9430 \times 10^{-2}$ \\
130 & $4.3458 \times 10^{-3}$ & $4.5934 \times 10^{-2}$ \\
140 & $4.3220 \times 10^{-3}$ & $4.2958 \times 10^{-2}$ \\
150 & $4.3029 \times 10^{-3}$ & $4.0398 \times 10^{-2}$ \\
\hline
\end{tabular}

exact and numerical solutions. In order to further substantiate the appositeness of the numerical scheme, we show in Figure 3(a) the exact and approximate solution curves at the control time $t=1$ on a single frame. The approximate solution appears to be very close to the exact one. In Figure 3(b), we plot the approximation error in log scales in $L^{2}$ and $H^{1}$ norms by varying the values of the spatial discretization step size $h$. The numerical estimated error is found to be in accordance with the theoretical estimate provided in Section 4 . Finally, in Table 1 , we present the convergence rates for $\wp_{1}$-elements by varying the spatial step size $h$. The results show that the convergence rate of the numerical scheme agrees with the theoretically estimated convergence rate in Section 4.

\subsection{Characteristic behavior of velocity profile}

Figures 4 and 5 are prepared to delineate the dependence of velocity profile on fractional exponents $\alpha$ and $\beta$. Plots are provided at fixed time instances $t=1$ and $t=5$. A strong effect of fractional exponents on velocity field has been demonstrated. It is observed in Figure 4 that the magnitude of the velocity field decreases with increasing values of $\alpha$. Moreover, this decrease is more rapid as $\alpha \rightarrow 1$. This indicates that the effect of an Oldroyd-B fluid rheology on the flow is much stronger in Brownian or ordinary models than in anomalous models. On the other hand, an increasing behavior of velocity amplitude is apparent 

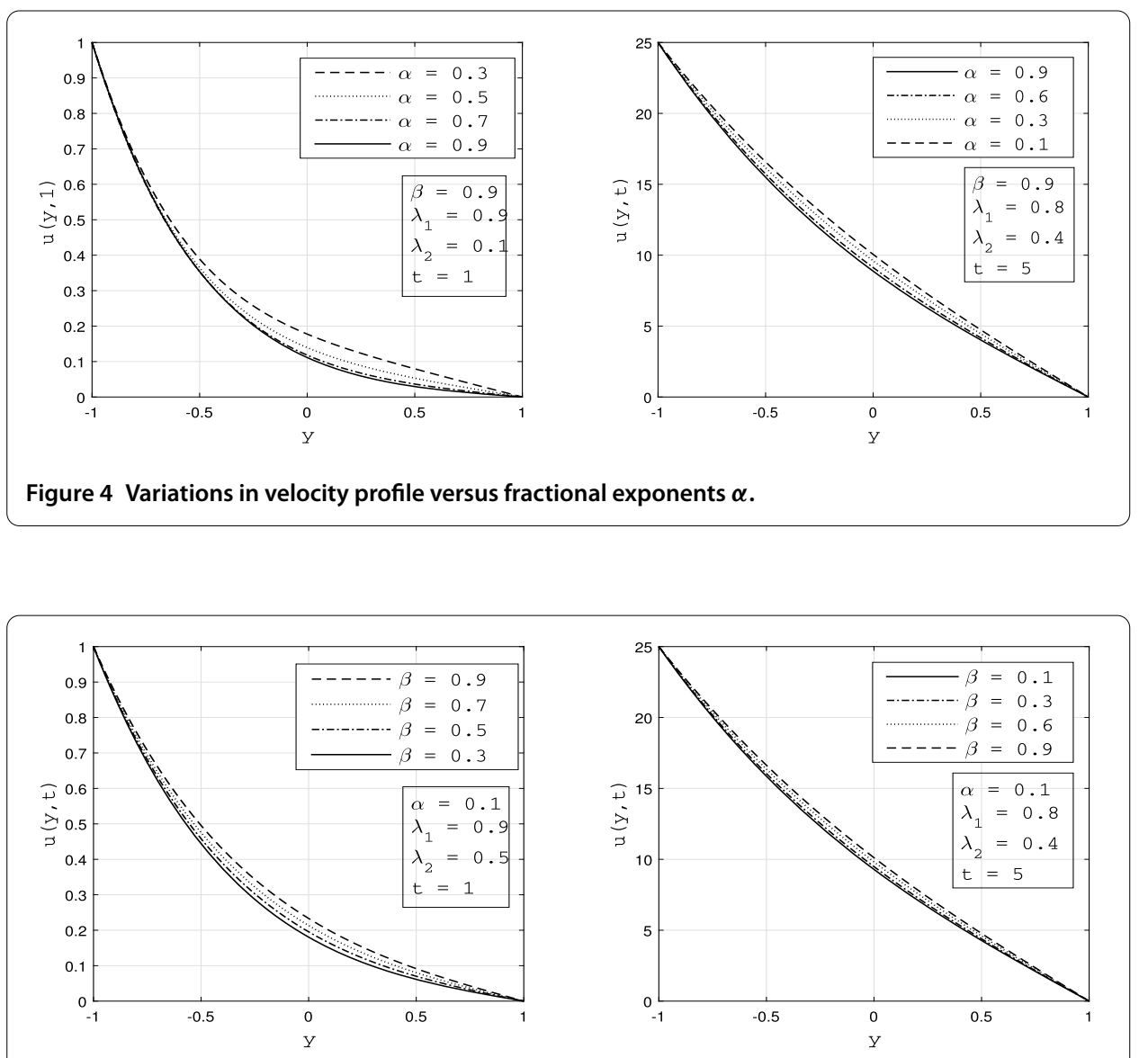

Figure 5 Variations in velocity profile versus fractional exponents $\beta$.

with increasing values of $\beta$ in Figure 5 in contrast with the case of $\alpha$. However, the dependence is certainly nonmonotonic in nature and cannot be generalized to other values of parameters, especially when $\alpha$ and $\beta$ are chosen to be very close. It is noted that $\alpha$ shows a shear-thinning behavior, whereas $\beta$ corresponds to shear-thickening behavior. Moreover, an increase in $\alpha$ reduces the boundary layer thickness, whereas $\beta$ shows an opposite trend on boundary layer thickness to that of $\alpha$. Based on these observations, wee can speculate that the fractional exponents in the anomalous Oldroyd-B model have strong effects on the velocity profile.

The effects of relaxation and retardation times on the velocity profile are presented in Figures 6 and 7. Different velocity curves at fixed times $t=1$ and $t=5$ are plotted for various choices of $\lambda_{1}, \lambda_{2}$, and the fractional exponents. Figure 6 shows that the magnitude of the velocity profile decreases with increasing values of $\lambda_{1}$. An opposite behavior is noted for $\lambda_{2}$ versus magnitude of the velocity profile in Figure 7. Moreover, $\lambda_{2}$ apparently has a stronger effect on velocity field than $\lambda_{1}$. The comparison shows that the velocity magnitude decays more slowly for large values of $\lambda_{1}$ and $\lambda_{2}$ than for small their values.

Finally, the transient velocity profile is depicted in Figure 8 for two different sets of rheological parameters and time intervals with anomalous behavior of the Oldroyd-B rheological model. 

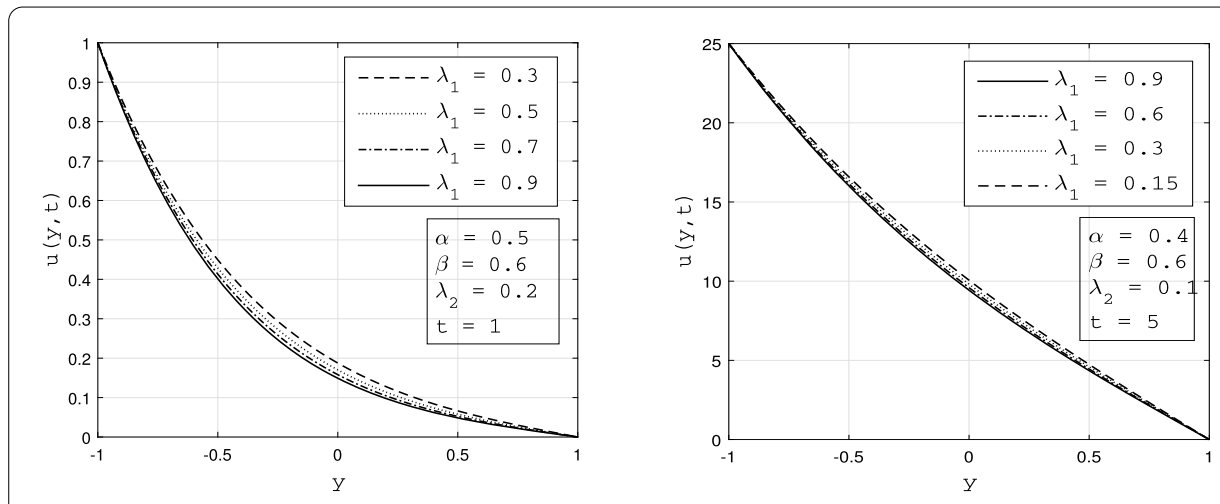

Figure 6 Variations in velocity profile versus relaxation time $\lambda_{1}$.
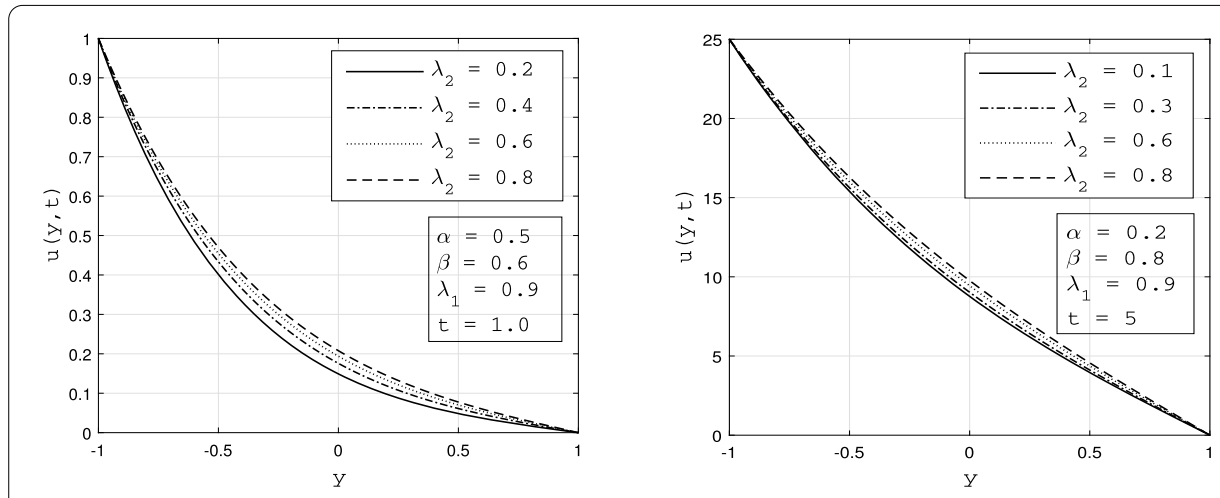

Figure 7 Variations in velocity profile versus retardation time $\lambda_{2}$.

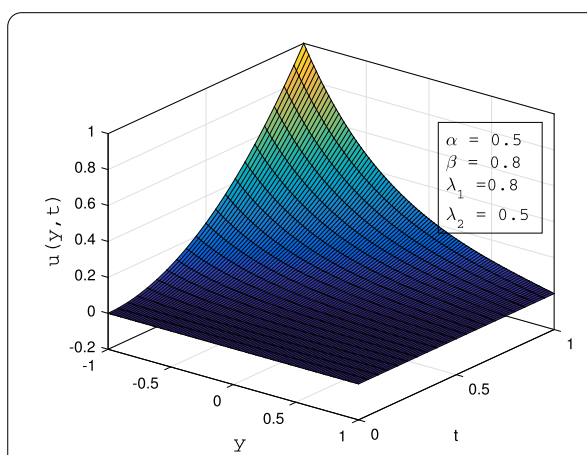

(a) $t \in[0,1]$.

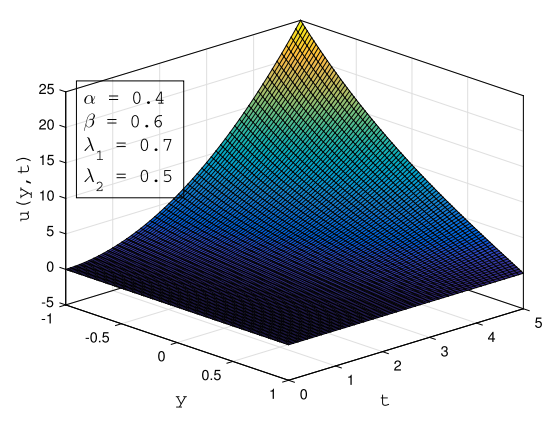

(b) $t \in[0,5]$.

Figure 8 Transient velocity profile.

\section{Concluding remarks}

In this article, we presented a Galerkin finite element method blended with a finite difference scheme for time fractional derivative to approximate flow velocity in an anomalous Oldroyd-B fluid confined between two infinite horizontal plates. The flow is induced by variable acceleration of the lower plate. No slip condition at the boundary is imposed. Convergence analysis of the numerical scheme is performed, and error bounds are pre- 
sented. Numerical results are discussed, and the influence of pertinent flow parameters on the velocity field is delineated. The results presented in this investigation generalize those for Brownian Oldroyd-B fluids and anomalous Maxwell fluids in analogous flow configurations. In the present study, the pressure gradient is considered to be negligible for simplicity. The results contained in this paper can be extended for Burgers fluids and will be discussed in a forthcoming investigation.

\section{Competing interests}

The authors declare that they have no competing interests.

\section{Authors' contributions}

All authors participated in drafting, revising, and commenting the manuscript. Also, all authors read and approved the final draft of manuscript.

\section{Author details}

'Department of Mathematics, Lahore University of Management Sciences, Opposite Sector U, DHA, Lahore, 54792 , Pakistan. ${ }^{2}$ Bio-imaging and Signal Processing Lab., Department of Bio and Brain Engineering, Korea Advanced Institute of Science and Technology, 291 Daehak-ro, Yuseong-gu, Daejeon, 34141, South Korea. ${ }^{3}$ Department of Mathematics Education, Kyungpook National University, 80 Daehak-ro, Buk-gu, Daegu, 41566, Korea. ${ }^{4}$ Department of Mathematics, COMSATS Institute of Information Technology, Park Road, Chak Shahzad, Islamabad, 44000, Pakistan.

\section{Acknowledgements}

This research was supported by the Korea Research Fellowship Program funded by the Ministry of Science, ICT and Future Planning through the National Research Foundation of Korea (NRF-2015H1D3A106240).

Received: 19 February 2016 Accepted: 2 September 2016 Published online: 13 September 2016

\section{References}

1. Richardson, SM: Flows of variable-viscosity fluids in ducts with heated walls. J. Non-Newton. Fluid Mech. 25, 137-156 (1987)

2. Lewis, RW, Nithiarasu, P, Seetharamu, KN: Fundamentals of the Finite Element Method for Heat and Fluid Flow. Wiley, Chichester (2004)

3. Massoudi, M, Phuoc, TX: Flow of a generalized second grade non-Newtonian fluid with variable viscosity. Contin. Mech. Thermodyn. 16, 529-538 (2004)

4. Khaled, ARA, Vafai, K: The role of porous media in modeling flow and heat transfer in biological tissues. Int. J. Heat Mass Transf. 46, 4989-5003 (2003)

5. Akbar, T, Nawaz, R, Kamran, M, Rasheed, A: Magnetohydrodynamic (MHD) flow analysis of second grade fluids in a porous medium with prescribed vorticity. AIP Adv. 5, 117133 (2015)

6. Rashidi, MM, Hayat, T, Erfani, E, Pour, SAM, Hendi, AA: Simultaneous effects of partial slip and thermal-diffusion and diffusion-thermo on steady MHD convective flow due to a rotating disk. Commun. Nonlinear Sci. Numer. Simul. 16(11), 4303-4317 (2011)

7. Liu, Y, Zheng, L, Zhang, X: Unsteady MHD Couette flow of a generalized Oldroyd-B fluid with fractional derivative. Comput. Math. Appl. 61, 443-450 (2011)

8. Liu, Y, Zheng, L, Zhang, X: The oscillating flows and heat transfer of a generalized Oldroyd-B fluid in magnetic field. IAENG Int. J. Appl. Math. 40, 276-281 (2010)

9. Fetecau, C, Athar, M, Fetecau, C: Unsteady flow of a generalized Maxwell fluid with fractional derivative due to a constantly accelerating plate. Comput. Math. Appl. 57(4), 596-603 (2009)

10. Kazem, S, Abbasbandy, S, Kumar, S: Fractional-order Legendre functions for solving fractional-order differential equations. Appl. Math. Model. 37, 5498-5510 (2013)

11. Hayat, T, Zaib, S, Asghar, S, Hendi, AA: Exact solutions in generalized Oldroyd-B fluid. Appl. Math. Mech. Engl. Ed. 33(4), 411-426 (2012)

12. Khan, M, Anjum, A, Fetecau, C, Qi, H: Exact solutions for some oscillating motions of a fractional Burgers' fluid. Math. Comput. Model. 51, 682-692 (2010)

13. Makris, N, Dargush, DF, Constantinou, MC: Dynamic analysis of generalized viscoelastic fluids. J. Eng. Mech. 119(8) 1663-1679 (1993)

14. Rasheed, A, Nawaz, R, Khan, SA, Hanif, H, Wahab, A: Numerical study of a thin film flow of fourth grade fluid. Int. J. Numer. Methods Heat Fluid Flow 25(4), 929-940 (2015)

15. Wahab, A, Rasheed, A, Nawaz, R, Javaid, N: Numerical study of two dimensional unsteady flow of an anomalous Maxwell fluid. Int. J. Numer. Methods Heat Fluid Flow 25(5), 1120-1137 (2015)

16. Wenchang, T, Wenxiao, P, Mingyu, X: A note on unsteady flows of a viscoelastic fluid with the fractional Maxwel model between two parallel plates. Int. J. Non-Linear Mech. 38, 645-650 (2003)

17. Xue, C, Nie, J, Tan, W: An exact solution of start-up flow for the fractional generalized Burgers' fluid in a porous half-space. Nonlinear Anal., Theory Methods Appl. 69(7), 2086-2094 (2008)

18. Bernard, JM: Weak and classical solutions of equations of motions for third grade fluids. ESAIM: Math. Model. Numer Anal. 33(6), 1091-1120 (1999)

19. Wu, YS, Qin, G: A generalized numerical approach for modeling multiphase flow and transport in fractured porous media. Commun. Comput. Phys. 6(1), 85-108 (2009) 
20. Oldroyd, JG: Non-Newtonian effects in steady motion of some idealized elastico-viscous liquids. Proc. R. Soc. Lond. A 245, 278-297 (1958)

21. Nield, DA, Bejan, A: Convection in Porous Media, 3rd edn. Springer, New York (2006)

22. Pathak, MG, Mulcahey, TI, Ghiaasiaan, SM: Conjugate heat transfer during oscillatory laminar flow in porous media. Int. J. Heat Mass Transf. 66, 23-30 (2013)

23. Heibig, A, Palade, Ll: On the rest state stability of an objective fractional derivative viscoelastic fluid model J. Math. Phys. 49, 043101 (2008)

24. Bird, RB, Armstrong, RC, Hassager, O: Dynamics of Polymeric Liquids. Wiley, New York (1987)

25. Truesdell, C, Noll, W: The Nonlinear Field Theories of Mechanics, 3rd edn. Springer, Berlin (2004)

26. Shehzad, SA, Abdullah, Z, Abbasi, FM, Hayat, T, Alsaedi, A: Magnetic field effect in three-dimensional flow of an Oldroyd-B nanofluid over a radiative surface. J. Magn. Magn. Mater. 399, 97-108 (2016)

27. Shehzad, SA, Alsaedi, A, Hayat, T, Alhuthali, MS: Thermophoresis particle deposition in mixed convection three-dimensional radiative flow of an Oldroyd-B fluid. J. Taiwan Inst. Chem. Eng. 45(3), 787-794 (2014)

28. Nadeem, S, UI Haq, R, Akbar, NS, Lee, C, Khan, ZH: Numerical study of boundary layer flow and heat transfer of Oldroyd-B nanofluid towards a stretching sheet. PLoS ONE 8(8), e69811 (2013)

29. Ramzan, M, Farooq, M, Alhothuali, MS, Malaikah, HM, Cui, W, Hayat, T: Three dimensional flow of an Oldroyd-B fluid with Newtonian heating. Int. J. Numer. Methods Heat Fluid Flow 25, 68-85 (2015)

30. Carcione, JM, Sanchez-Sesma, FJ, Luzón, F, Gavilán, JJP: Theory and simulation of time-fractional fluid diffusion in porous media. J. Phys. A, Math. Theor. 46, 345501 (2013)

31. Hernández-Jiménez, A, Hernández-Santiago, J, Macias-García, A, Sánchez-González, J: Relaxation modulus in PMMA and PTFE fitting by fractional Maxwell model. Polym. Test. 21, 325-331 (2002)

32. Bagley, RL, Torvik, PT: A theoretical basis for the application of fractional calculus to viscoelasticity. J. Rheol. 27(3), 201-210 (1983)

33. Bagley, RL, Torvik, PT: On the fractional calculus model of viscoelastic behavior. J. Rheol. 30(1), 133-155 (1986)

34. Heibig, A, Palade, Ll: Well posedness of a linearized fractional derivative fluid model. J. Math. Anal. Appl. 380(1), 188-203 (2011)

35. Song, DY, Jiang, TQ: Study on the constitutive equation with fractional derivative for the viscoelastics fluids-modified Jeffreys model and its application. Rheol. Acta 37, 512-517 (1998)

36. Lipscombe, TC: Comment on 'Application of the homotopy method for analytical solution of non-Newtonian channel flows'. Phys. Scr. 81, 037001 (2010)

37. Sajid, M, Hayat, T, Asghar, S: Comparison of the HAM and HPM solutions of thin film flows of non-Newtonian fluids on a moving belt. Nonlinear Dyn. 50, 27-35 (2007)

38. Pletcher, RH, Tannehill, JC, Anderson, D: Computational Fluid Mechanics and Heat Transfer. CRC Press, Boca Raton (2011)

39. Reddy, JN, Gartling, DK: The Finite Element Method in Heat Transfer and Fluid Dynamics, 3rd edn. CRC Press, Boca Raton (2010)

40. Zhuang, P, Liu, Q: Numerical method of Rayleigh-Stokes problem for heated generalized second grade fluid with fractional derivative. Appl. Math. Mech. Engl. Ed. 30(12), 1533-1546 (2009)

41. Lin, $Y, X u, C:$ Finite difference/spectral approximations for the time-fractional diffusion equation. J. Comput. Phys. 225 1533-1552 (2007)

42. Li, C, Zhao, Z, Chen, Y: Numerical approximation of nonlinear fractional differential equations with subdiffusion and superdiffusion. Comput. Math. Appl. 62, 855-875 (2011)

43. Jiang, Y, Ma, J: High-order finite element methods for time-fractional partial differential equations. J. Comput. Appl. Math. 235, 3285-3290 (2011)

44. Podlubny, I: Fractional Differential Equations. Academic Press, San Diego (1999)

45. Kilbas, AA, Srivastava, HM, Trujillo, JJ: Theory and Applications of Fractional Differential Equations. Elsevier, Amsterdam (2006)

46. Toms, BA, Strawbridge, DJ: Elastic and viscous properties of dilute solutions of polymethyl methacrylate in organic liquids. Trans. Faraday Soc. 49, 1225-1232 (1953)

47. Adams, RA: Sobolev Spaces. Academic Press, New York (1975)

48. Ciarlet, PG: The Finite Element Methods for Elliptic Problems. Classics in Applied Mathematics. SIAM, Philadelphia (2002)

49. Thomée, V: Galerkin Finite Element Methods for Parabolic Problems. Springer, Berlin (2006)

\section{Submit your manuscript to a SpringerOpen ${ }^{\ominus}$ journal and benefit from:}

- Convenient online submission

Rigorous peer review

- Immediate publication on acceptance

- Open access: articles freely available online

- High visibility within the field

- Retaining the copyright to your article 